\title{
Identifying and Ranking Sources of SSR Based on the Concept of Subsynchronous
} Power

Gao, Bo; Wang, Yang; Xu, Wilsun; Yang, Guangya

Published in:

IEEE Transactions on Power Delivery

Link to article, DOI:

10.1109/TPWRD.2019.2916848

Publication date:

2020

Document Version

Peer reviewed version

Link back to DTU Orbit

Citation (APA):

Gao, B., Wang, Y., Xu, W., \& Yang, G. (2020). Identifying and Ranking Sources of SSR Based on the Concept of Subsynchronous Power. IEEE Transactions on Power Delivery, 35(1), 258 - 268.

https://doi.org/10.1109/TPWRD.2019.2916848

\section{General rights}

Copyright and moral rights for the publications made accessible in the public portal are retained by the authors and/or other copyright owners and it is a condition of accessing publications that users recognise and abide by the legal requirements associated with these rights.

- Users may download and print one copy of any publication from the public portal for the purpose of private study or research.

- You may not further distribute the material or use it for any profit-making activity or commercial gain

- You may freely distribute the URL identifying the publication in the public portal 


\title{
Identifying and Ranking Sources of SSR Based on the Concept of Subsynchronous Power
}

\author{
Bo Gao, Student Member, IEEE, Yang Wang, Member, IEEE, Wilsun Xu, Fellow, IEEE, \\ and Guangya Yang, Senior Member, IEEE
}

\begin{abstract}
This paper presents a measurement-based method for real-time determination of the wind farms or wind turbines that contribute most significantly to a subsynchronous resonance (SSR) event. The method is based on the concept of subsynchronous power (SSP). This paper has shown it is the SSP that drives the SSR. By monitoring the direction and amount of SSPs produced by different wind farms or turbines, the most critical wind farms or turbines can be identified and quantified. A dq0 transform based algorithm is developed to calculate the SSP in real time. The method overcomes the interference of fundamental frequency components and non-steady-state SSR components on the extraction of the SSP component. Several test cases have demonstrated the usefulness of the proposed concept and method.
\end{abstract}

Index Terms - Subsynchronous resonance (SSR), wind farm, subsynchronous power, SSR source detection.

\section{INTRODUCTION}

$I^{1}$ $\mathrm{T}$ is well known that the series compensated wind farm systems are vulnerable to subsynchronous resonance (SSR) due to the interaction between the series capacitor and the doublyfed induction generator (DFIG) [1]. Several SSR events have been reported in wind farms of USA and China [2]-[4]. During these incidents, SSR became so strong that it caused the tripping of a large number of wind turbines (WTs) or even damaged some of the generators' crowbar circuits.

A wind-farm supplied power system usually consists of multiple geographically distributed wind farms and each wind farm contains different numbers and types of WTs. As a result, wind farms respond differently during an SSR event. It is thus of great importance to identify the critical wind farms or individual WTs that have significant contributions to an SSR event. This problem may be called the "SSR source detection" problem. Appropriate actions can be taken only after the SSR sources are identified correctly and timely.

SSR source detection can be potentially achieved by using the model-based methods. For example, the state-space based small signal analysis [5]-[8] can calculate participation factors of wind farms. Another method is the impedance based analysis [4], [9]-[12]. This method establishes equivalent impedances for the wind farms and then analyzes the system stability based

B. Gao and W. Xu are with the Department of Electrical and Computer Engineering, University of Alberta, Alberta, Canada (e-mail: bgao1@ualberta.ca andwxu@ualberta.ca).

Y. Wang is currently with the College of Electrical Engineering and Information Technology, Sichuan University, Chengdu, Sichuan, 610017, China (e- on impedance-based criterion. All these methods require detailed data regarding internal components, control parameters, and the structures of the wind generators. Since generator manufacturers are reluctant to provide controller data, such methods are difficult to apply with confidence [14]. Furthermore, the accuracy of the results is also affected by the power output of each wind turbine. The outputs can be different even for turbines within a wind farm. Consequently, they cannot be used to predict or determine SSR sources reliably. In summary, such tools are more suitable for offline based scenario studies.

The above concern leads to the development of measurement-based methods. However, our extensive literature review found that very limited effort has been devoted in this area. Only one related work is found in [14]. Although not clearly stated, the paper tries to use the measured resistance of the wind farms to determine whether it is contributing to the negative damping. Unfortunately, the non-steady-state nature of SSR current and voltage imposes challenges on Fourier-transform based impedance estimation.

Subsynchronous frequency is essentially an interharmonic frequency. The problem of SSR source detection can therefore be approached from the perspective of interharmonic source detection. Based on this reasoning and the work of [15], this paper proposes to use the power generated by various wind farms or turbines at the subsynchronous frequency, called subsynchronous power (SSP), as an indicator to rank critical wind farms or turbines. An algorithm for SSP calculation using real-time measurements taken at the interconnection points of wind farms or wind turbines is developed for the purpose.

The remainder of this paper is organized as follows. Section II explains the source of SSR from the perspective of power generation at the subsynchronous frequency and introduces the concept of subsynchronous power. Section III presents an algorithm for online measurement and monitoring of subsynchronous power. Sensitivity studies are also conducted to show its robustness. In section IV, case studies are conducted to validate the usefulness of the proposed method for SSR source detection and ranking.

\section{THE CONCEPT OF SUBSYNCHRONOUS POWER}

This section explains the idea of SSP and uses a simple circuit to illustrate its characteristics. The challenges of measuring

mail: fwang@scu.edu.cn)

G. Yang is with the Centre for Electric Power and Energy (CEE), Technical University of Denmark, 2800 Kgs. Lyngby, Denmark (gyy@elektro.dtu.dk) 
SSP are then discussed.

\section{A. Mechanism of Subsynchronous Power Generation}

Fig. 1 depicts a simple DFIG-based wind farm connected to the grid through a series compensated line. The system has a natural resonance frequency due to the series resonance between the series capacitor and the combined inductance of the line and the wind farm. The resonance frequency could be lower than the fundamental frequency of the system, i.e. it is at the subsynchronous frequency. If the series circuit has a positive resistance overall at that frequency, the resonance is damped and the system can operate normally.

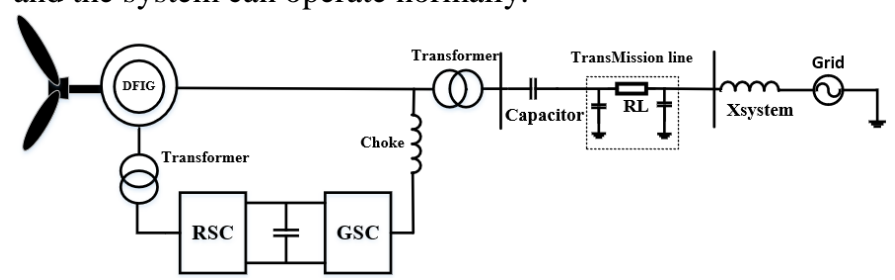

Fig. 1 A DFIG-based wind farm connected to the system with series compensation

It is well known that an induction generator produces power by behaving as a negative rotor resistance at the fundamental frequency. This negative resistance is caused by the negative slip. Similarly, the slip with respective to the subsynchrnous frequency in a DFIG is also negative. Thus, the rotor resistance can be negative at that frequency as well. If this frequency coincides with the series resonance frequency mentioned earlier and the overall circuit has a net negative resistance, the resonance could be amplified. A subsynchronous resonance is produced.

There is another way to explain the above phenomenon: the rotor produces a power at the subsynchronous frequency. This power is defined as the SSP in this paper. It is the SSP that drives and sustains the subsynchronous resonance. Thus, we can monitor the SSPs produced by various wind farms or individual DFIGs to identify the sources of SSR and to determine which wind farms or DFIGs contribute the most to an SSR event. This reasoning is also applicable to cases where the controllers of wind generators cause the SSR. It has been reported that poorly designed controllers can contribute to the negative resistance, leading to a more severe SSR phenomenon [16].

Interharmonic power has been used successfully to identify interharmonic sources [15]. The proposed SSP concept is inspired by the interharmonic source detection research but there are two main differences: (1) the interharmonics are steadystate phenomena while an SSR is a dynamic phenomenon which involves non-steady-state (i.e. growing) voltage and current waveforms. As a result, steady-state based interharmonic source detection method cannot be applied here directly. (2) A system typically has one interharmonic source at the interharmonic frequency of concern. There could be multiple SSR sources in a system. There is, therefore, a need to identify and rank the sources according to their impacts to an SSR event.

\section{B. Characteristics of SSP}

A simple third order system shown in Fig. 2 is adopted to illustrate the concept and characteristics of SSP. In the figure, the two parallel branches represent two DFIG-based wind turbines. One branch has a negative resistance and the other has a positive resistance. To replicate an SSR phenomenon, the system parameters are selected to yield the following two features:

- The system has a positive resistance under fundamental frequency $\left(\omega_{0}\right)$, namely, the system is stable at this frequency.

- The system experiences an amplified oscillation at a subsynchronous frequency, denoted as $\omega_{s s r}$. At this frequency, the equivalent resistance of the whole circuit is negative.

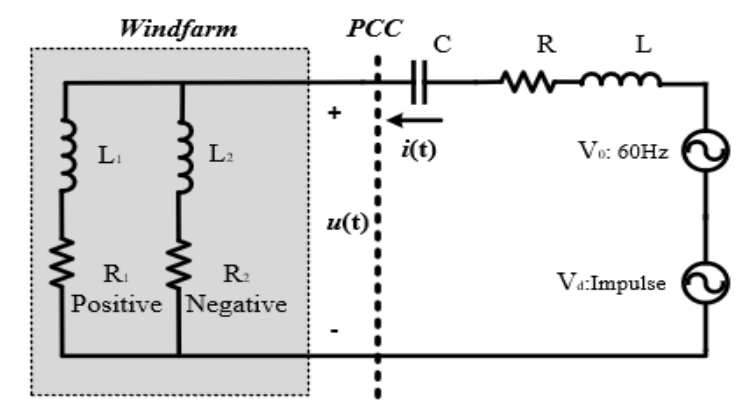

Fig. 2 Studied third order system

Table I shows one set of parameters meeting the above requirements. The SSR frequency of the circuit can be determined as $31.63 \mathrm{~Hz}$ and the damping ratio is -0.54 . In the simulation, the SSR event is triggered by an impulse voltage source $\mathrm{V}_{\text {impulse. }}$ This source contains all frequency components, so it is used to mimic system disturbances that can excite an SSR event. The circuit response is shown in Fig. 3. It can be seen that the current waveform measured at the PCC is modulated by a growing component of SSR frequency. The response of the instantaneous power [defined as $v(t) \times i(t)$ ] is more complex since it contains more than one modulation frequencies.

Table I

Parameters of studied third order system

\begin{tabular}{cccccccc}
\hline $\mathrm{L}$ & $\mathrm{C}$ & $\mathrm{R}$ & $\mathrm{R} 1$ & $\mathrm{~L} 1$ & $\mathrm{R} 2$ & $\mathrm{~L} 2$ & $\mathrm{~V} 0$ \\
\hline $0.2 \mathrm{H}$ & $100 \mu \mathrm{F}$ & $1 \Omega$ & $60 \Omega$ & $0.02 \mathrm{H}$ & $-3 \Omega$ & $0.05 \mathrm{H}$ & $1000 \mathrm{~V}$ \\
\hline
\end{tabular}

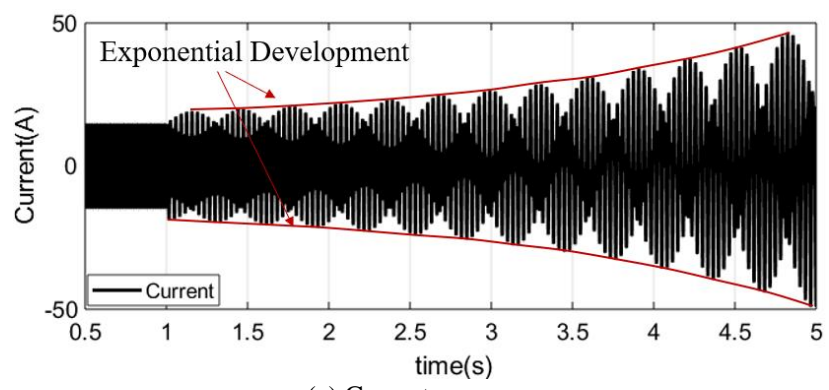

(a) Current response

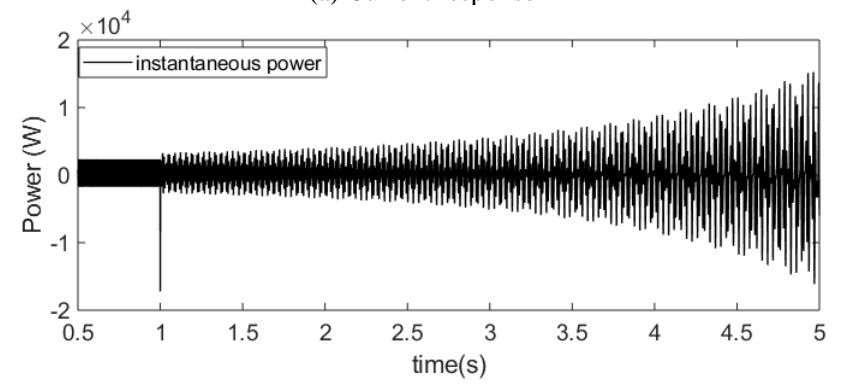

(b) Power response

Fig. 3 Circuit response of the studied third order system 
Circuit analysis can show that the voltage and current at the PCC contain three components as shown in (1), where $I_{0}, V_{0}$ are the magnitudes of fundamental current and voltage, respectively. $I_{\mathrm{ssr}}, V_{\mathrm{ssr}}$ are the magnitudes of SSR current and voltage, respectively. $\theta_{0}, \delta_{0}$ are the phase angles of fundamental current and voltage, respectively. $\theta, \delta$ are the phase angles of SSR current and voltage, respectively. $\alpha$ is the damping ratio of the SSR component. $I_{d}$ and $V_{d}$ are the DC current and voltage, and $\alpha_{d}$ represents the corresponding damping ratio.

$$
\begin{aligned}
& i(t)=I_{0} \cos \left(\omega_{0} t+\theta_{0}\right)+I_{s s r} e^{-a t} \cos \left(\omega_{s s} t+\theta\right)+I_{d} e^{-\alpha_{d} t} \\
& v(t)=V_{0} \cos \left(\omega_{0} t+\delta_{0}\right)+V_{s s r} e^{-a t} \cos \left(\omega_{s s r} t+\delta\right)+V_{d} e^{-\alpha_{d} t}
\end{aligned}
$$

Since the DC components decay rapidly $\left(\alpha_{d}=961.7\right)$, the expression of the instantaneous power can be established by ignoring the DC terms, as shown in (2).

$$
\begin{aligned}
& p(t)=i(t) v(t) \\
& \approx V_{0} I_{0} \frac{\cos \left(2 \omega_{0} t+\theta_{0}+\delta_{0}\right)+\cos \left(\theta_{0}-\delta_{0}\right)}{2}+ \\
& I_{0} V_{s s r} e^{-a t} \frac{\cos \left(\left(\omega_{0}+\omega_{s s r}\right) t+\theta_{0}+\delta\right)+\cos \left(\left(\omega_{0}-\omega_{s s r}\right) t+\theta_{0}-\delta\right)}{2}+ \\
& V_{0} I_{s s r} e^{-a t} \frac{\cos \left(\left(\omega_{0}+\omega_{s s r}\right) t+\delta_{0}+\theta\right)+\cos \left(\left(\omega_{0}-\omega_{s s r}\right) t+\delta_{0}-\theta\right)}{2}+ \\
& V_{s s r} I_{s s r} e^{-2 a t} \frac{\cos \left(2 \omega_{s s r} t+\theta+\delta\right)+\cos (\theta-\delta)}{2}
\end{aligned}
$$

As seen from (2), there are four terms in the instantaneous power expression. The first term is the fundamental frequency power (with a frequency of $2 \omega_{0}$ ). This term has no relationship with the SSR event. The second and third terms represent the interaction between the SSR components and the fundamental frequency components (with frequencies of $\omega_{0}-\omega_{\text {ssr }}$ and $\left.\omega_{0}+\omega_{\text {ssr }}\right)$. It can be shown that the average powers associated with these two terms can be either positive or negative. The polarities are strongly affected by starting time of the SSR event, i.e. $\theta$ and $\delta$. Therefore, they do not contain consistent information on the characteristics of SSR. The fourth term is clearly related to the SSR voltage and current. Following the definition of the active (i.e. average) power at fundamental frequency, the average value of this term over the period of SSR frequency is

$$
\begin{aligned}
P_{s s r}(t) & =\frac{\omega_{s s r}}{2 \pi} \int_{t}^{t+\frac{2 \pi}{\omega_{s s r}}} v_{s s r}(t) i_{s s r}(t) d t \\
& =\frac{\omega_{s s r}}{4 \pi} V_{s s r} I_{s s r} e^{-2 \alpha t}\left(e^{-4 \pi \alpha / \omega_{s s r}}-1\right)\left(\frac{1}{-2 a} \cos (\theta-\delta)+\right. \\
& \left.\frac{-a \cos \left(2 \omega_{s s r} t+\theta+\delta\right)+\omega_{s s r} \sin \left(2 \omega_{s s r} t+\theta+\delta\right)}{2\left(a^{2}+\omega_{s s r}{ }^{2}\right)}\right)
\end{aligned}
$$

As expected, the above power is not a constant. This is due to the non-steady-state nature of the SSR phenomenon. (If an SSR event were in steady-state, $\alpha=0$, then $P_{s s r}$ would become a constant). We propose to remove the sinusoidal component (with frequency of $2 \omega_{s s r}$ ) with one more level of averaging over half of the SSR period, i.e. $\pi / \omega_{s s r}$, as follows

$$
\overline{P_{s s r}\left(t_{k}\right)}=\left(P_{s s r}\left(t_{k}\right)+P_{s s r}\left(t_{k-1}\right)+\ldots+P_{s s r}\left(t_{k-\frac{\pi}{\omega_{s s r}} n_{s+1}}\right)\right) /\left(\frac{\pi}{\omega_{s s r}} n_{s}\right)
$$

where, $k$ stands for the $\mathrm{k}^{\text {th }}$ sample of the raw voltage or current waveform data and $n_{s}$ is the sampling rate. This operation leads to the following (moving) average power:

$$
P_{s s p}(t)=\overline{P_{s s r}(t)}=\frac{\omega_{s s r}}{-8 \pi \alpha} V_{s s r} I_{s s r} e^{-2 \alpha t}\left(e^{-4 \pi \alpha \omega_{s r}}-1\right) \cos (\theta-\delta)
$$

The above power is the defined SSP in this paper. Fig. 4 shows the trend of SSP obtained based on (5) and circuit simulation results. The results are highly consistent except there is a transient response in simulated SSP. The reason is that the simulation result includes the DC component of (1). Negative power in the figure means power is injected to the series branch. The defined SSP in Fig. 4 can represent SSP of an individual or collective of same wind turbines.

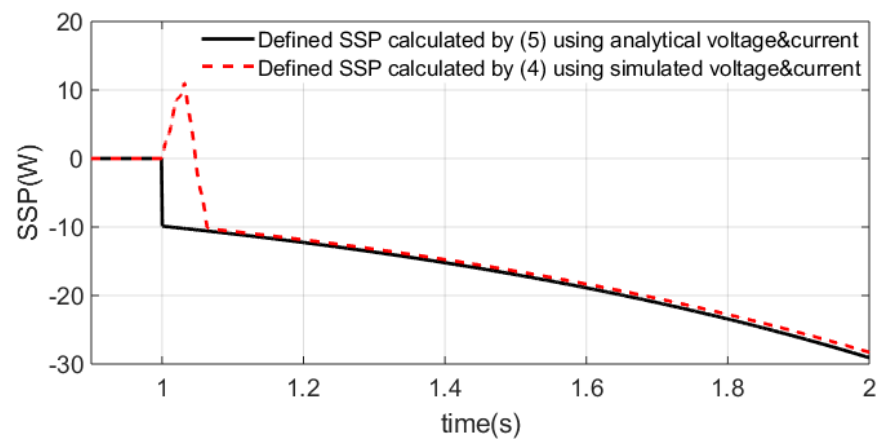

Fig. 4 Trend of subsynchronous power.

In the following, we will show that the above definition can capture the main characteristics of the power that drives the unstable SSR in the circuit of Fig. 2. Here we examine the energy stored in the series capacitor since this capacitor makes the system resonant. From $t$ to $t+\Delta T$, the energy stored in the capacitor can be determined as

$$
\begin{aligned}
E(t)= & \int_{t}^{t+\Delta T} p_{c}(t) d t=\int_{t}^{t+\Delta T} u_{c} * C \frac{d u_{c}}{d t} d t=\frac{C}{2}\left[u_{c}^{2}(t+\Delta T)-u_{c}^{2}(t)\right] \\
= & \frac{C V_{c}^{2}}{2}\left\{\cos ^{2}\left[\omega_{0}(\mathrm{t}+\Delta \mathrm{T})+\delta_{c}\right]-\cos ^{2}\left(\omega_{0} \mathrm{t}+\delta_{c}\right)\right\} \\
& +C V_{c s s r} V_{c} \mathrm{e}^{-a t}\left\{\cos \left[\omega_{0}(\mathrm{t}+\Delta \mathrm{T})+\delta_{c}\right] \cos \left[\omega_{s s r}(\mathrm{t}+\Delta \mathrm{T})+\delta_{c s s r}\right] e^{-a \Delta T}\right. \\
& \left.-\cos \left(\omega_{0} \mathrm{t}+\delta_{c}\right) \cos \left(\omega_{s s r} \mathrm{t}+\delta_{c s s r}\right)\right\} \\
& +\frac{C V_{c s s r}{ }^{2} \mathrm{e}^{-2 a t}}{2}\left\{\cos ^{2}\left[\omega_{s s r}(\mathrm{t}+\Delta \mathrm{T})+\delta_{c s s r}\right] e^{-2 \alpha \Delta T}-\cos ^{2}\left(\omega_{s s r} \mathrm{t}+\delta_{c s s r}\right)\right\}
\end{aligned}
$$

where, " $u_{c}$ " stands for capacitor voltage. By selecting $\Delta T$ as the least common multiple of the fundamental period and SSR period, the energy expression in (6) can be rewritten as

$$
\begin{aligned}
& E(t)=C V_{c s s r} V_{c} \mathrm{e}^{-a t}\left(e^{-a \Delta T}-1\right) \frac{\cos \left(\omega_{0} t+\delta_{c}\right) \cos \left(\omega_{s s r} t+\delta_{c s s r}\right)}{2} \cos ^{2}\left(\omega_{s s r} t+\delta_{c s s r}\right)\left(e^{-2 \alpha \Delta T}-1\right) \\
& +\frac{C V_{c s s r}^{2} \mathrm{e}^{-2 a t}}{2}
\end{aligned}
$$

The above expression shows that $E(t)$ has two components: (1) Component that grows at a rate of $e^{-a t}$ which is the $1^{\text {st }}$ term. The coefficient of this term (the underlined items) can be either positive or negative depending on the angles. It means that this term does not lead to the accumulation of energy in the capacitor. (2) Component that grows at a rate of $e^{-2 a t}$, i.e. the $2^{\text {nd }}$ term. This component always grows in one direction due to the $\cos ^{2}(x)$ 
characteristic. As a result, energy builds up in the capacitor. This is clearly the unstable component that is directly related to SSR. Since the SSP defined earlier also grows at the rate of $e^{-}$ 2at, we can conclude that the proposed SSP concept is able to capture the nature of the SSR phenomenon. Of course, there are many power/energy components with different frequencies in an actual SSR event. The above analysis indicates that the SSP defined here can represent reasonably well the main power component that leads to an SSR event.

A further justification for the validity of the SSP definition is the following: the frequency of oscillation of instantaneous power is twice of the frequency of the current and voltage producing that power. Since the SSR voltage and current have a frequency of $\omega_{s s}$. Their corresponding power component ought to have a frequency of $2 \omega_{s s}$. The SSP defined earlier is the only component that has a frequency of $2 \omega_{s s r}$ among the four components shown in (2).

Although the above discussions are based on an example circuit, the analysis relies on the voltage and current at the PCC and the capacitor only regardless the circuit components. Therefore, the conclusions of this section have general applicability to actual power systems.

\section{Challenges of measuring SSP}

Accordingly, if the SSP can be measured in real time, it is possible to locate the driver (i.e. the source) for an SSR event. However, the SSP component cannot be easily extracted due to the following real-time challenges:

- The only measurable component is the instantaneous power $p(t)$ (i.e. calculated from $i$ and $v$ ). Since the SSR frequency is not known a prior so an average over an SSR period cannot be done. In addition, there is no guarantee that such an average can eliminate components of other frequencies;

- The SSR voltage and current have exponential components, so FFT cannot be used to extract them accurately for SSP calculation. Even if the exponential component is small and the waveform can be considered as quasi steady-state (i.e. interharmonics), a long data window is needed for estimating the subsynchronous frequency [17]. It is also difficult to determine the window length in practice.

- The SSR voltage and current might be extracted by using a low pass filter (LPF) in time domain. However, the wide range of possible SSR frequencies makes it difficult to design an LPF with guaranteed performance. The exponential component could corrupt the results as well.

- The Prony method may extract the parameters of the SSR voltage and current. SSP can then be calculated based on the results. This is an indirect method and it is relatively slow for real-time implementation. The method can also introduce larger errors if the SSR component is relatively small such as at the early stage of an SSR event.

There is therefore a need for a fast and easy to implement method for direct calculation of SSP based on measured voltage and current waveforms. This is the subject of the next section.

\section{Method to Extract Subsynchronous Power}

In an SSR event, three phase currents and voltages can be described as
$v_{a}(t)=V_{0} \cos \left(\omega_{0} t+\delta_{0}\right)+V_{s s r} e^{-a t} \cos \left(\omega_{s s t} t+\delta\right)$

$v_{b}(t)=V_{0} \cos \left(\omega_{0} t+\delta_{0}-120^{\circ}\right)+V_{s s r} e^{-a t} \cos \left(\omega_{s s r} t+\delta-120^{\circ}\right)$

$v_{c}(t)=V_{0} \cos \left(\omega_{0} t+\delta_{0}+120^{\circ}\right)+V_{s s r} e^{-a t} \cos \left(\omega_{s s r} t+\delta+120^{\circ}\right)$

$i_{a}(t)=I_{0} \cos \left(\omega_{0} t+\theta_{0}\right)+I_{s s r} e^{-a t} \cos \left(\omega_{s s t} t+\theta\right)$

$i_{b}(t)=I_{0} \cos \left(\omega_{0} t+\theta_{0}-120^{\circ}\right)+I_{s s r} e^{-a t} \cos \left(\omega_{s s r} t+\theta-120^{\circ}\right)$

$i_{c}(t)=I_{0} \cos \left(\omega_{0} t+\theta_{0}+120^{\circ}\right)+I_{s s r} e^{-a t} \cos \left(\omega_{s s r} t+\theta+120^{\circ}\right)$

where, $a, b$ and $c$ represent the phase sequence. It should be noted that there may be supersynchronous components during SSR events. However, this paper focus on the SSR caused by the interaction between DFIGs and series-compensated systems. For such case, the supersynchronous components are negligible as the phenomenon is mainly induced by the induction generator effect (IGE).

\section{A. DQ frame based SSP extraction}

This paper proposes to use $d q$ transformation to facilitate the SSP extraction. A $d q$ frame is selected to be synchronous with the fundamental frequency. Thus, $d q$ transformation can be described as (9).

$$
[F]_{d q}=\frac{2}{3} T[F]_{a b c}
$$

where

$T=\left[\begin{array}{ccc}\cos \left(\omega_{0} t\right) & \cos \left(\omega_{0} t-120^{\circ}\right) & \cos \left(\omega_{0} t+120^{\circ}\right) \\ -\sin \left(\omega_{0} t\right) & -\sin \left(\omega_{0} t-120^{\circ}\right) & -\sin \left(\omega_{0} t+120^{\circ}\right)\end{array}\right]$

and $[F]_{a b c}$ denotes the voltages or currents in $a b c$ frame. $[F]_{d q}$ denotes the voltages or currents in $d q$ frame. Substituting (8) into (9), we can obtain

$$
\begin{aligned}
& i_{d}(t)=I_{0} \cos \theta_{0}+I_{s s r} e^{-a t} \cos \left(\omega_{0} t-\omega_{s s r} t-\theta\right) \\
& i_{q}(t)=I_{0} \sin \theta_{0}-I_{s s r} e^{-a t} \sin \left(\omega_{0} t-\omega_{s s r} t-\theta\right) \\
& v_{d}(t)=V_{0} \cos \delta_{0}+V_{s s r} e^{-a t} \cos \left(\omega_{0} t-\omega_{s s r} t-\delta\right) \\
& v_{q}(t)=V_{0} \sin \delta_{0}-V_{s s r} e^{-a t} \sin \left(\omega_{0} t-\omega_{s s r} t-\delta\right)
\end{aligned}
$$

The active power $P(t)$ including both fundamental frequency components and SSR frequency components can be then calculated by applying the classic active power calculation equation in $d q$ frame, as shown in (15).

$$
\begin{aligned}
P(t) & =\frac{3}{2}\left(i_{d}(t) v_{d}(t)+i_{q}(t) v_{q}(t)\right) \\
& =\frac{3}{2} V_{0} I_{0} \cos \left(\theta_{0}-\delta_{0}\right)+\frac{3}{2} e^{-a t}\left(I_{0} V_{s s r} \cos \left(\omega_{0} t+\theta_{0}-\omega_{s s t} t-\delta\right)\right. \\
& \left.+V_{0} I_{s s r} \cos \left(\omega_{0} t+\delta_{0}-\omega_{s s t} t-\theta\right)\right)+\frac{3}{2} V_{s s r} I_{s s r} e^{-2 a t} \cos (\theta-\delta)
\end{aligned}
$$

The terms in (15) are similar with those in (2), except the former one represents the three-phase power while the latter one represents the single-phase power. Integrating the fourth term in (15) over the SSR period leads to (16). It can be found that (16) is exactly same with the defined SSP. 


$$
\begin{aligned}
P_{s s r}(t) & =\frac{\omega_{s s r}}{2 \pi} \int_{t}^{t+\frac{2 \pi}{\omega_{s s r}}} \frac{3}{2} V_{s s r} I_{s s r} e^{-2 a t} \cos (\theta-\delta) d t \\
& =\frac{3 \omega_{s s r}}{-8 \pi a} V_{s s r} I_{s s r} e^{-2 \alpha t}\left(e^{-4 \pi \alpha / \omega_{s s r}}-1\right) \cos (\theta-\delta)
\end{aligned}
$$

To isolate the fourth term in (15), the first three terms need to be removed from $P(t)$. It is noticeable that four terms in (15) can be classified into two categories: 1) AC power which includes the second and third terms and 2) DC power which includes the first term (fundamental frequency power) and the fourth term (SSP). Since the practical SSR frequency is rarely close to the fundamental frequency, the frequency of the $\mathrm{AC}$ power i.e. $\omega_{0}-\omega_{\text {ssr }}$ is much greater than zero. Thus, it is practically feasible to design a low pass filter to remove the $\mathrm{AC}$ power. In terms of the fundamental frequency power, its value is expected to be unchanged at the early stage of the SSR event [3], hence this component can be eliminated by subtracting the DC power before the SSR event. Fig. 5 compares the SSP of the wind farm in Fig. 2 calculated by its definition in (5) and by the proposed method to extract fourth term in (15). The system is changed to three-phase with the same parameters in each phase, in which (5) still holds for SSP in each phase. It can be observed that there is a small difference between the defined SSP and the SSP obtained by the proposed algorithm. This difference is caused by the delay of the LPF. To validate this inference, the SSP defined in (4) is processed with a same LPF. It can be seen that there is a good agreement between the defined SSP with filter delay and that calculated by the proposed algorithm.

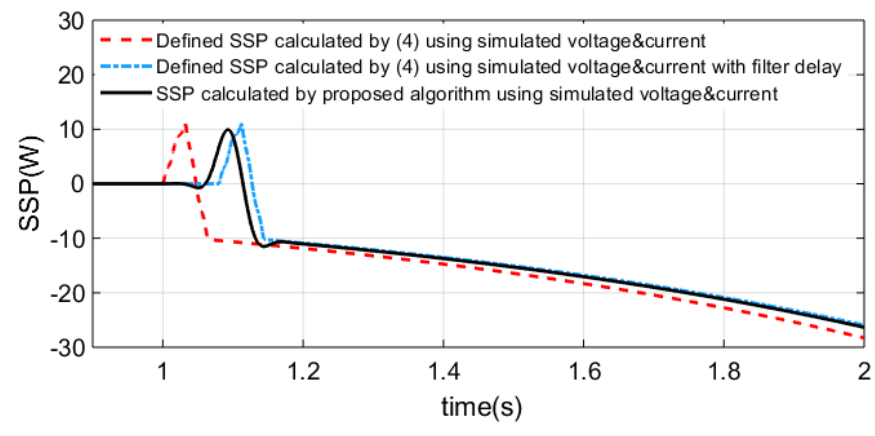

Fig. 5 SSP calculated based on the proposed algorithm and definition.

The above derivation assumes the SSR current and voltage are purely positive sequence. In practice, the SSR components may contain small part of negative sequence quantities. By following the same process, it can be shown that the negative sequence SSR components would lead to similar results. The only difference is that the frequency of the AC power becomes $\omega_{0}+\omega$. As the frequency $\omega_{0}+\omega$ is higher than $\omega_{0}-\omega$, this component can be more easily filtered by the low pass filter. Thus, the proposed algorithm can extract the SSP at both the positive sequence and the negative sequence simultaneously.

\section{B. Practical Considerations}

This subsection investigates the impact of harmonic distortions, the low pass filter design and fundamental frequency power variations on the proposed method.

\section{1) Impact of harmonic distortions}

The real-life power system contains harmonics. It would be straightforward to eliminate the impact of harmonics by using an additional LPF on the raw data at the beginning stage. However, such a solution induces additional delays. In the following analysis, it will be shown that the proposed algorithm is in fact robust against system harmonic distortions. By including a positive-sequence harmonic component with the frequency of $\omega_{h}$, the voltage and current waveforms in (8) can be rewritten as (17). Only phase A quantities are shown due to the limited space.

$$
\begin{aligned}
& v_{a}(t)=V_{0} \cos \left(\omega_{0} t+\delta_{0}\right)+V_{s s r} e^{-a t} \cos \left(\omega_{s s r} t+\delta\right)+V_{h} \cos \left(\omega_{h} t+\delta_{h}\right) \\
& i_{a}(t)=I_{0} \cos \left(\omega_{0} t+\theta_{0}\right)+I_{s s r} e^{-a t} \cos \left(\omega_{s s r} t+\theta\right)+I_{h} \cos \left(\omega_{h} t+\theta_{h}\right)
\end{aligned}
$$

where $V_{h}$ and $I_{h}$ represent the magnitudes of harmonic voltage and current respectively, $\delta_{\mathrm{h}}$ and $\theta_{h}$ represent the phase angles of harmonic voltage and current respectively. Current and voltage in $d q$ frame can be computed by the transformation in (9). The active power is then derived as follows

$$
\begin{aligned}
& P(t)=\frac{3}{2}\left(i_{d}(t) v_{d}(t)+i_{q}(t) v_{q}(t)\right) \\
& =\frac{3}{2} V_{0} I_{0} \cos \left(\theta_{0}-\delta_{0}\right)+\frac{3}{2} I_{h} V_{h} \cos \left(\theta_{h}-\delta_{h}\right)+\frac{3}{2} e^{-2 a t} I_{s s r} V_{s s r} \cos (\theta-\delta) \\
& +\frac{3}{2} e^{-a t}\left(I_{0} V_{s s r} \cos \left(\left(\omega_{0}-\omega_{s s r}\right) t+\theta_{0}-\delta\right)+V_{0} I_{s s r} \cos \left(\left(\omega_{0}-\omega_{s s r}\right) t+\delta_{0}-\theta\right)\right) \\
& +\frac{3}{2}\left(I_{h} V_{0} \cos \left(\left(\omega_{h}-\omega_{0}\right) t+\theta_{h}-\delta_{0}\right)+I_{0} V_{h} \cos \left(\left(\omega_{h}-\omega_{0}\right) t-\theta_{0}+\delta_{h}\right)\right) \\
& +\frac{3}{2} e^{-a t}\left(I_{h} V_{s s r} \cos \left(\left(\omega_{h}-\omega_{s s r}\right) t+\theta_{h}-\delta\right)+I_{s s r} V_{h} \cos \left(\left(\omega_{h}-\omega_{s s r}\right) t+\delta_{h}-\theta\right)\right)
\end{aligned}
$$

Similar as (15), terms in (18) also can be classified into AC power and DC power. The AC power in (18) consists of sinusoid signals with three frequencies: $\omega_{h}-\omega_{0}, \omega_{h}-\omega_{s s r}$, and $\omega_{0}-\omega_{s s r}$. Since the low pass filter is designed for filtering the components with the frequency of $\omega_{0}-\omega_{s s r}$, its performance can be guaranteed at higher frequencies i.e. $\omega_{h}-\omega_{0}$ and $\omega_{h}-\omega_{s s r}$. The DC power in (18) includes the fundamental frequency power, the harmonic power and the SSP. Since the harmonic power is also a steady-state power, it can be removed by subtracting the DC power measured before the SSR event. Therefore, the harmonic distortion is expected to have limited impact on the proposed method. Fig. 6 shows the calculated SSP under different system harmonic distortions. It can be seen that the results are consistent regardless of the system distortion level. Note that if the harmonic component is in negative sequence, $\omega_{h}-\omega_{0}$ and $\omega_{h}-\omega_{s s r}$ in (18) should be replaced by $\omega_{h}+\omega_{0}$ and $\omega_{h}+\omega_{s s r}$, respectively. The results are similar as the AC power still can be filtered by the low pass filter.

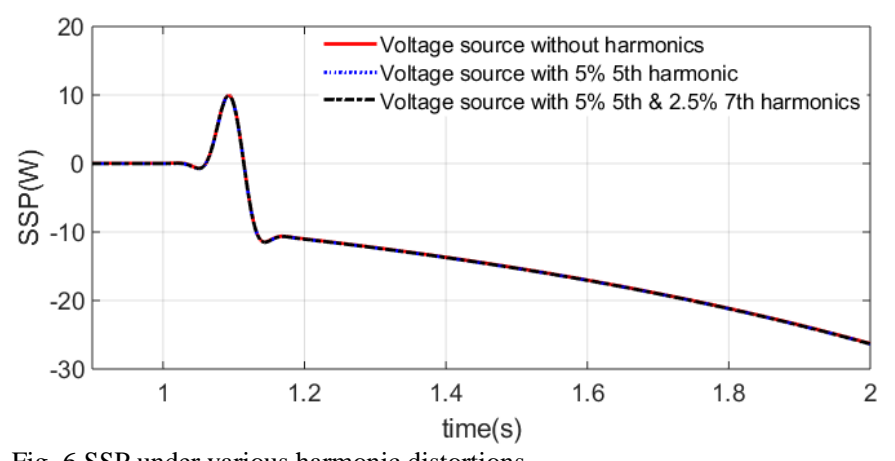

Fig. 6 SSP under various harmonic distortions 


\section{2) Low pass filter}

In the proposed method, a low pass filter is needed to filter the AC power with the lowest frequency of $\omega_{0}-\omega$. Since the proposed method does not determine the value of $\omega$, the filter should be pre-designed for general SSR events.

In practice, the SSR frequency is expected to be lower than $45 \mathrm{~Hz}$ (for a $60 \mathrm{~Hz}$ system) [6]. According to real-life SSR events in recent years, the reported SSR frequencies are between $6 \sim 8 \mathrm{~Hz}$ in China [4] and around $22 \mathrm{~Hz}$ in ERCOT of US [2]. Thus, a FIR LPF (Equiripple) specified with a pass band edge of $15 \mathrm{~Hz}$ and stopband edge of $25 \mathrm{~Hz}$ is selected in this paper. The passband ripple is set as 0.1 while the stopband attenuation is 80 . It should be mentioned that using the LPF causes certain transient response and time delay, which can be seen in Fig. 5Fig. 6. The time delay of the LPF used is around $150 \mathrm{~ms}$.

\section{3) Variation of fundamental frequency power}

In the previous illustration, the fundamental frequency power is assumed to be unchanged after SSR occurrence. However, this assumption does not always hold in practice, as the fundamental frequency power may vary, for example due to the tripping of WTs. A statistical method is further proposed below to address this issue.

During an SSR event, the DC power extracted by dq transform can be described as:

$$
P_{D C}(t)=P_{0}+P_{s s r} e^{-\alpha t}
$$

where, $P_{0}$ represents the steady state power including the fundamental frequency power and the harmonic power, and $P_{s s} e^{-\alpha t}$ is the SSP. Assuming $P_{0}$ does not change for consecutive three points, we can obtain

$$
\begin{aligned}
& P_{D C}(t)=P_{0}+P_{s s r} e^{-\alpha t} \\
& P_{D C}(t-\Delta t)=P_{0}+P_{s s r} e^{-\alpha(t-\Delta t)} \\
& P_{D C}(t-2 \Delta t)=P_{0}+P_{s s r} e^{-\alpha(t-2 \Delta t)}
\end{aligned}
$$

where, $\Delta t$ represents the time interval. Solving the above equation leads to

$$
e^{\alpha \Delta t}=\frac{P_{D C}(t-\Delta t)-P_{D C}(t-2 \Delta t)}{P_{D C}(t)-P_{D C}(t-\Delta t)}
$$

For a certain short period such as $30 \Delta t$ (4ms for a sampling rate of $7680 \mathrm{~Hz}$ ), if all calculated $e^{\alpha \Delta t}$ are roughly equal which can be checked by (22), $P_{D C}$ can be then considered to follow the formulation in (19). In other words, $P_{0}$ is roughly constant and the calculated SSP can be trusted. Otherwise, the calculated SSP should be discarded.

$$
\operatorname{abs}\left(\frac{\max \left(e^{\alpha \Delta t}\right)-\min \left(e^{\alpha \Delta t}\right)}{\operatorname{avg}\left(e^{\alpha \Delta t}\right)}\right)<1.2
$$

A simulation study has been conducted to validate the effectiveness of the above method by using the third-order circuit in Fig. 2. A variation in fundamental frequency power is created by increasing the fundamental frequency voltage to 1.5 times of its previous value at $3 \mathrm{~s}$. The SSP calculated with and without the statistical check are illustrated in Fig. 7. For comparison, the real SSP is obtained by removing the fundamental frequency source in the third-order circuit. As can be seen from Fig. 7(a), the result without the statistical check experiences large errors when the fundamental frequency power varies. By using the proposed statistical check, the SSP can always be tracked accurately. Note no result is provided around 3s in Fig. 7(b) as the change of the fundamental frequency power makes (22) invalid. Since the fundamental frequency power is unlikely to change all the time, such a short time of blank has no significant impact on the SSR source detection.

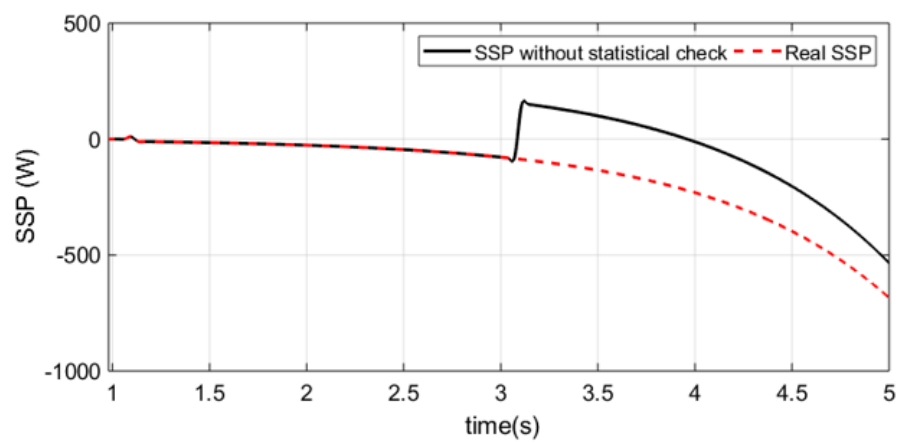

(a)

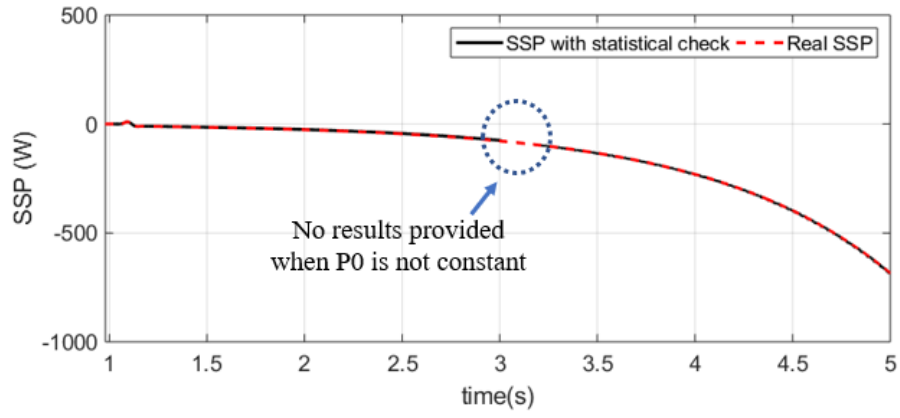

(b)

Fig. 7 SSP results by base method and statistical check method

\section{CASe Studies}

This section demonstrates the usefulness of the proposed algorithm in SSR source detection by using the modified IEEE first benchmark model.

\section{A. System Description}

The studied system is shown in Fig. 8. There are three wind farms connected to the point of coupling (PCC) by collectors. The length (impedance) of collectors may vary due to different locations of the wind farms. Among three wind farms, wind farm1 (WF1) is PMSG based, while wind farm 2 (WF2) and wind farm 3 (WF3) are DFIG based. Rated power for single PMSG and DFIG are $2 \mathrm{MW}$ and $1.5 \mathrm{MW}$ respectively. The parameters of the collectors and transmission lines can be found in [12], [17].

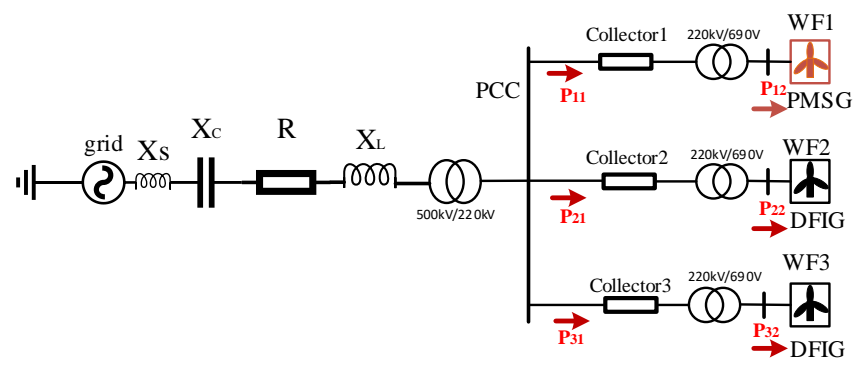

Fig. 8 System diagram in simulation 


\section{B. Modelling of Wind Farms}

Modelling of DFIG and PMSG has been well documented in previous publications [18]-[21]. Fig. 9 shows the typical structure of a 1.5MW DFIG, where a sixth-order model of induction generator is used, with a two-mass drive model to represent the generator shaft. An inductance at the entrance of the grid side converter is used to eliminate the switching frequency distortions, and one shunt $\mathrm{RC}$ filter is also added for the same purpose. The control schemes of rotor side converter (RSC) and grid side converter (GSC) are illustrated in Fig. 9(b) and Fig. 9(c), respectively. The outer loop of RSC is used to control the active power $\left(P_{s}\right)$ and the reactive power $\left(Q_{s}\right)$ of the generator, while the outer control loop GSC is used to control the DC link voltage $\left(V_{d c}\right)$ and the reactive power (generally set as 0 ). Proportional-integrate (PI) controller is used in these control loops.

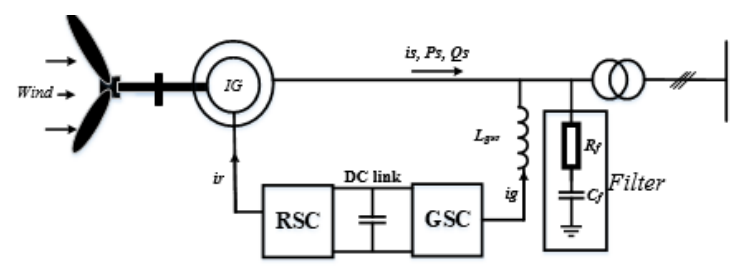

(a)

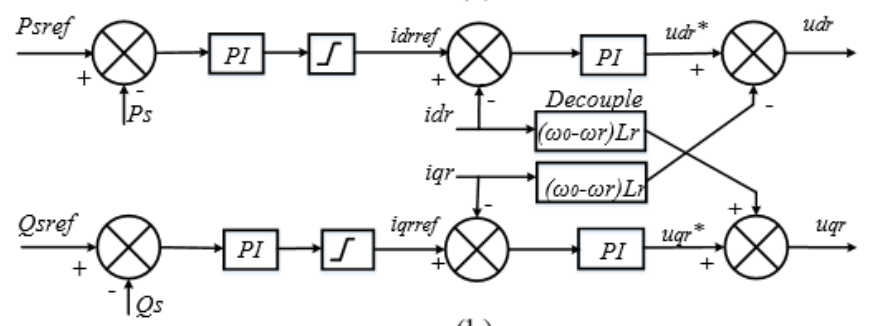

(b)

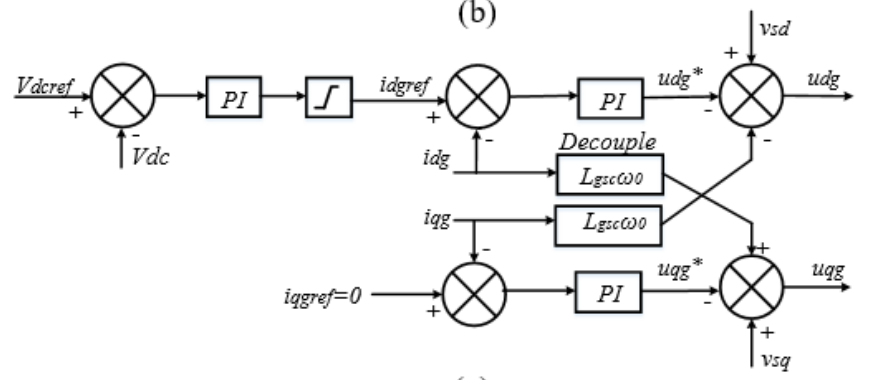

(c)

Fig. 9 DFIG-based windfarm. (a) System structure, (b) Control block diagram of RSC, (c) Control block diagram of GSC. * subscript " $d$ " means d axis, " $q$ " means q axis, and "ref' means reference value.

The typical structure of a PMSG-based windfarm is designed as Fig. 10(a), where a wind turbine with a PMSG is connected with the grid through back-to-back converters. There are two converters for controlling purpose: machine side converter (MSC) controls the generator side while the grid side converter (GSC) is designed to control the grid side. It is well known that GSC is more critical for dynamic analysis, and its control scheme is illustrated in Fig. 10(b). The outer control loops of GSC control the DC link voltage and the reactive power $\left(Q_{g}\right)$ to be constant by using PI controllers. An LC filter is added at the entrance of the grid side converter to filter out high frequency distortions induced by the converter.

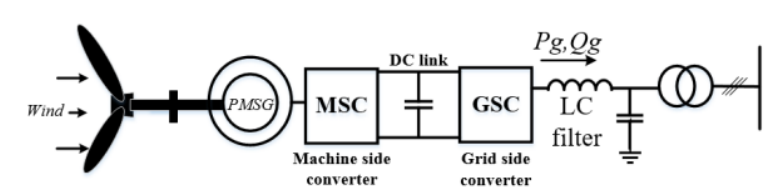

(a)

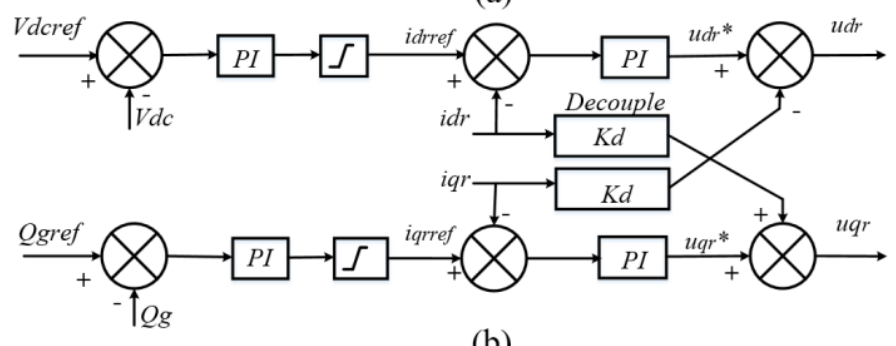

(b)

Fig. 10 PMSG-based windfarm. (a) Structure of PMSG-based windfarm, (b) GSC control. * subscript " $d$ " means d axis, " $q$ " means q axis, and "ref" means reference value.

\section{Critical Wind Farm Identification}

Two case studies are conducted to validate the effectiveness of the proposed algorithm for identifying the critical wind farms in SSR events. In both cases, SSPs at two different locations are measured for each wind farm. One is the SSP measured at the terminals of wind farms, denoted as $\mathrm{P}_{12}, \mathrm{P}_{22}$, and $\mathrm{P}_{32}$ in Fig. 8 . The other is the SSP measured at the PCC, denoted as $\mathrm{P}_{11}, \mathrm{P}_{21}$, and $\mathrm{P}_{31}$ in Fig. 8.

\section{1) Case 1}

The system condition of case 1 is described as follows. The wind speed for all three wind farms is $9 \mathrm{~m} / \mathrm{s}$. The number of wind turbines in service in WF1 WF2 and WF3 are all set as 100, 200 and 200, respectively. The capacitor is switched from $280 \mu \mathrm{F}$ to $175 \mu \mathrm{F}$ (compensation level 9.3\%) at 10 second in simulation to cause an unstable oscillation in the system. The collectors of three wind farms have different lengths: collectors of WF1 and WF3 are 50km, and the collector of WF2 is $190 \mathrm{~km}$. Because of the same number of the wind turbines and the same wind speed for WF2 and WF3, this case is designed to reveal the impact of the collectors on the SSP generation.

The simulation results are depicted in Fig. 11. It can be observed that all measured SSPs are amplifying, indicating an unstable SSR event. According to Fig. 8, the SSP at the terminals of WF2 and WF3 are negative while the SSP at the terminal of WF1 is positive. The absorbed SSP by WF1 is found to be much smaller than the amount produced by WF2 and WF3. This is due to the large positive resistance of the PMSG based wind farm. The result is in accordance with the previous finding that the DFIG based wind farm is the main cause of the SSR event, while the PMSG based wind farm is normally not involved [14].

In terms of the SSP measured at the PCC, $\mathrm{P}_{31}$ of WF3 is still negative while $\mathrm{P}_{21}$ of WF2 turns to be positive. The difference lies on the different power consumption of collectors. P31 stays negative indicates collector3 cannot completely dissipate the WF3-generated SSP, thus this branch still injects SSP to the system. On the other hand, the longer collector 2 has a stronger ability to consume SSP due to its larger resistance. The positive P21 indicates the WF2-genearated SSP has been completely dissipated by collector2. As a result, the branch containing WF2 and collector2 is absorbing SSP instead of producing SSP. 


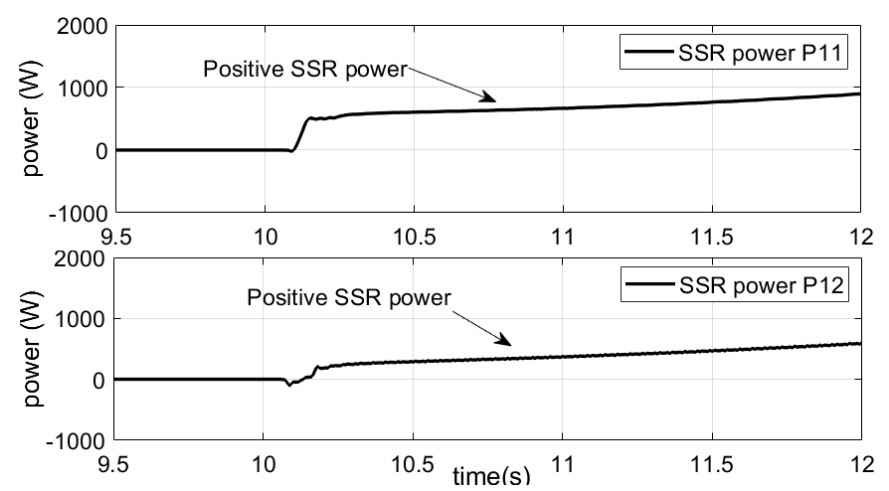

(a) SSP measured at WF1
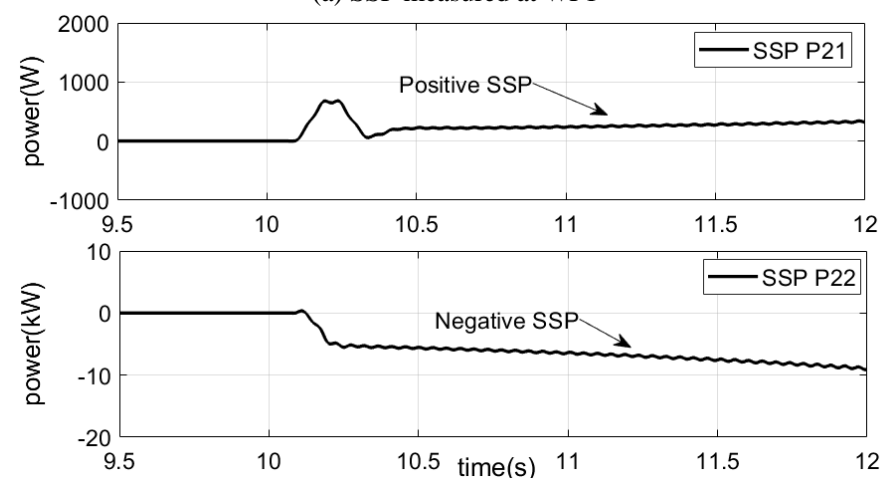

(b) SSP measured at WF2
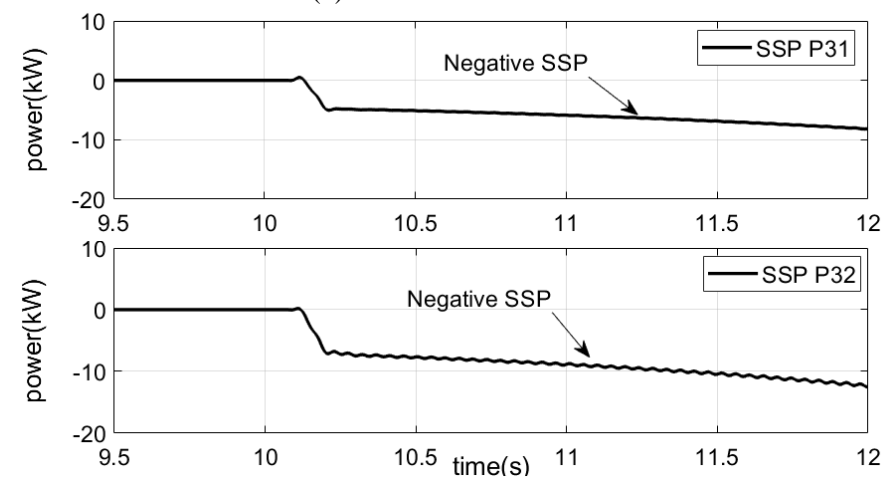

(c) SSP measured at WF3

Fig. 11 Measured SSP for different wind farm branches in case 1

From the analysis above, it can be seen that although DFIGs generate SSPs at the SSR frequency, the entire wind farm may not contribute to the SSR phenomenon if the impact of the collector is considered. The real-time measured SSP is found to be an effective index to indicate the participation of a wind farm. In this case, WF3 is identified as the root cause of the SSR event, while WF2 is actually not involved. Such a result can significantly simplify the troubleshooting process in post-SSR analysis and find the critical wind farms in which countermeasures may apply.

\section{2) Case 2}

The system condition of case 2 is described as follows. The collectors for all three wind farms are set to be $130 \mathrm{~km}$. The numbers of the wind turbines of WF1, WF2 and WF3 are set as 100,300 , and 100 , respectively. The wind speed is same with that in case 1 . The compensation level of the transmission line increases to $11.2 \%(145 \mu \mathrm{F})$ and is switched from $285 \mu \mathrm{F}$ at 10 second in the simulation. Since the lengths of collectors and wind speed are same for three wind farms, this case is designed to reveal the impact of the number of wind turbines in service on the SSP generation.

According to simulation results, WF1 in this case still absorbs SSPs at two measurement locations, thus only SSPs of WF2 and WF3 are depicted in Fig. 12 for further analysis. The results are similar with those in case 1 . WF3 is found to produce SSPs at both its terminal and the end of its collector. On the other hand, WF2 generates SSP at its terminal, but absorbs SSP at the end of its collector. The difference originates from the number of wind turbines in operation. Due to the larger number of DFIGs online, the negative resistance of WF2 is smaller. As a result, WF2 generates less SSP which can be absorbed by collector2. On the contrary, the SSP generated by WF3 exceeds the dissipating ability of collector3. Therefore, in this case, WF3 is still the only contributor to the SSR event. The result, again, indicates that it is not rigorous to identify the root cause of the SSR event simply based on the type of the wind farm.

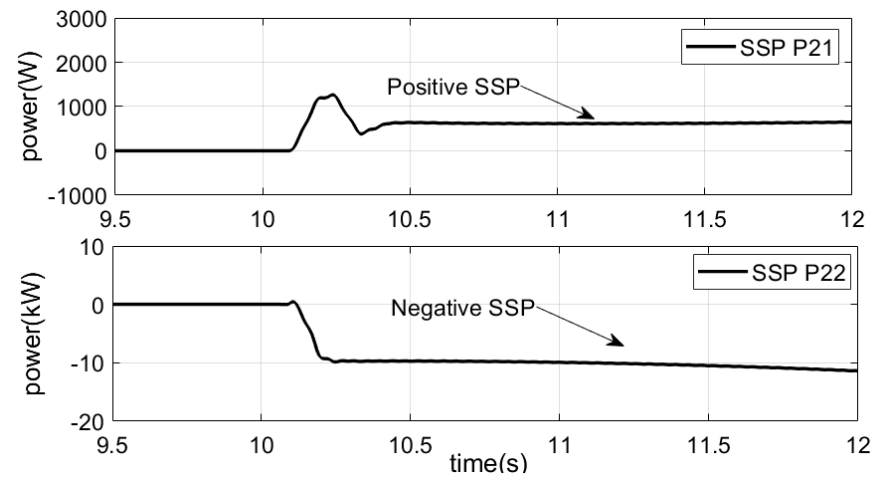

(a) SSP measured at WF2
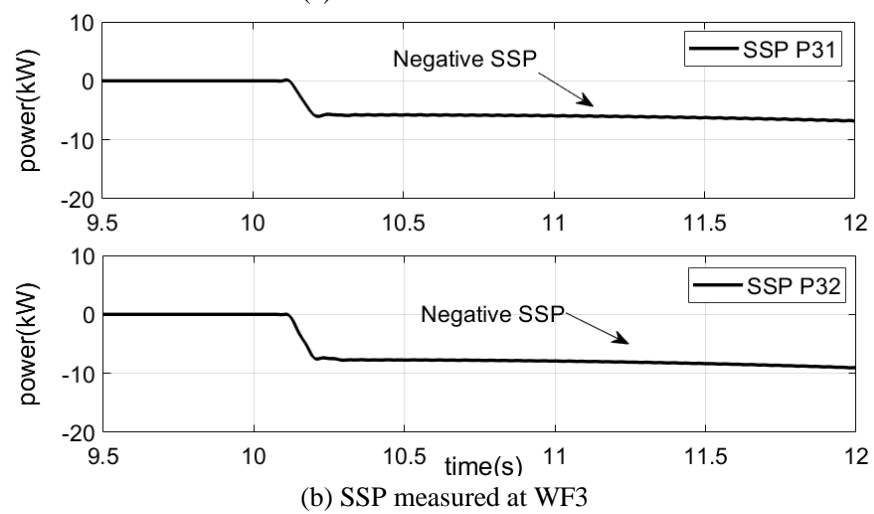

Fig. 12 Measured SSP for different wind farm branches in case 2

\section{Ranking Responsibilities}

In the previous two cases, only one wind farm, WF3, contributes to the SSR event. A more general situation is that multiple wind farms inject SSPs to the system simultaneously. For such case, it is necessary to rank the contribution of each wind farm. An additional case is studied here to show the application of SSP for responsibility assignment. In this case, the SSPs of WF2 and WF3 measured at the PCC are both negative. This is achieved by changing the number of the wind turbines of WF1, WF2 and WF3 in case 2 to 100, 200 and 100 respectively, and setting all three collectors as $100 \mathrm{~km}$. 
Fig. 13 depicts the SSPs measured at the PCC for WF2 and WF3. It can be observed that although the SSPs of both wind farms are negative, their magnitudes are different. For the same instant, WF3 always produces more SSP than WF2. In this paper, the wind farm with a larger magnitude of the SSP at the PCC corresponds to a larger contribution to the SSR severity. Thus, WF3 should be ranked as the critical wind farm that contributes most to the SSR event. The validity of the contribution ranking on basis of SSP can be explained by the RLC circuit shown in Fig. 14(a). In the figure, SSPs generated by multiple wind farms pass through the PCC into the system and are partially consumed by the resistance on the system side $\left(R_{1}\right)$. The rest of SSPs is equally alternated between the capacitor and the equivalent inductance of the whole system (mainly line inductor), as shown in Fig. 14 (b). Instead of being dissipated timely, the SSPs will be cumulatively stored in the capacitor. As for capacitor, larger stored power means larger current or voltage, which indicates a more severe SSR incident. Therefore, it is reasonable to define the SSP of a wind farm as its contribution to the incident.

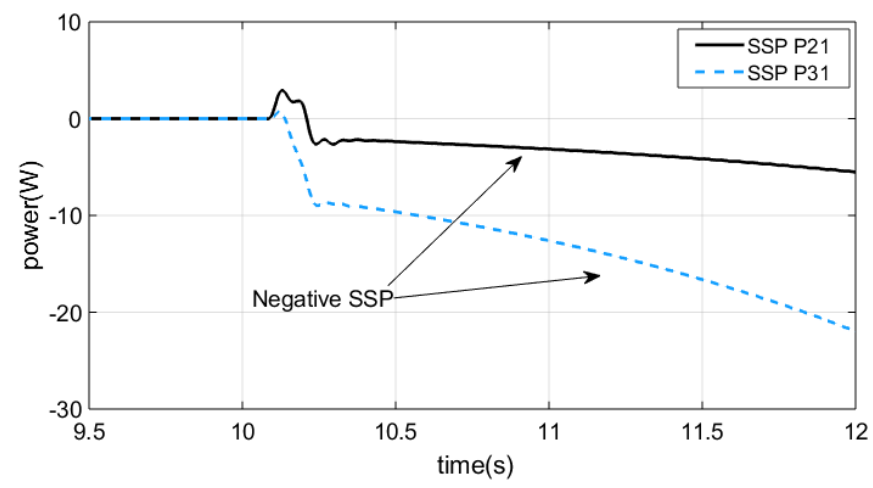

Fig. 13 Measured SSP for different wind farm branches

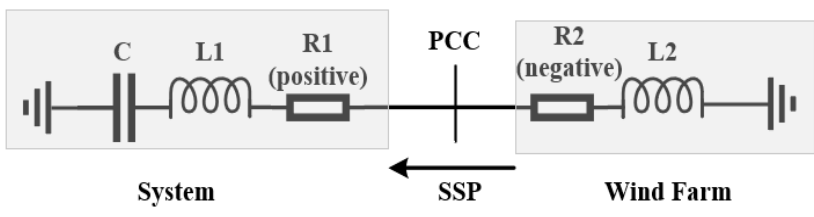

(a)

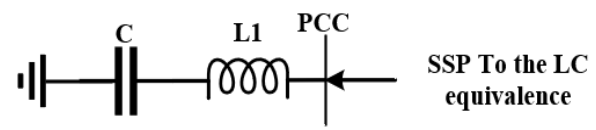

(b)

Fig. 14 RLC equivalence of system under SSR frequency

Above case studies are conducted in windfarms containing only one kind of wind turbines. All wind turbines in one windfarm are thus considered to have same contributions. To determine the contribution of certain wind turbines or a group of wind turbines in windfarms containing different kinds of wind turbines, the method can be implemented by adding SSP monitoring at various terminals of wind turbines, collector points or windfarms. As long as a monitor shows positive SSP output, the monitored downstream is a contributor to the SSR.

\section{CONCLUSION}

This paper has presented a new and effective method for SSR source detection. The basic idea is to use the subsyncrhonous power generated by various wind farms or wind turbines as an indicator to rank their impacts to the SSR event. Main contributions of this paper are summarized as follows.

1) Introduction of the concept of subsynchronous power (SSP). Through mathematical analysis, this work has shown that it is the SSP that drives and sustains the SSR phenomenon. This concept thus enables the SSR analysis from the perspective of power generation.

2) A novel dq0 transform-based algorithm that makes SSP measurable. The proposed algorithm directly extracts the SSP from the measured voltage and current waveforms. The knowledge of the SSR frequency is not needed. In addition, the algorithm is easy to implement and requires small computation burden. Thus, it can be easily added into modern power meters for real-time measurement.

3) Demonstration of the proposed concept and its application in SSR source detection. Case studies with different scenarios have been conducted to demonstrate the usefulness of the proposed method. Sensitivity studies have also been performed to show its robustness.

\section{REFERENCES}

[1] "Reader's guide to subsynchronous resonance," in IEEE Transactions on Power Systems, vol. 7, no. 1, pp. 150-157, Feb. 1992.

[2] J. Adams, V. A. Pappu, and A. Dixit, "ERCOT experience screening for Sub-Synchronous Control Interaction in the vicinity of series capacitor banks," in Proc. IEEE PES General Meeting, 2012, pp.1-5.

[3] L. Wang, X. Xie, Q. Jiang, H. Liu, Y. Li, and H. K. Liu, "Investigation of SSR in practical DFIG-based wind farms connected to a series-compensated power system," IEEE Trans. Power Syst., vol. 30, no. 5, pp. 27722779, Sept. 2015.

[4] X. Xie, X. Zhang, H. Liu, H. Liu, Y. Li and C. Zhang, "Characteristic Analysis of Subsynchronous Resonance in Practical Wind Farms Connected to Series-Compensated Transmissions," in IEEE Transactions on Energy Conversion, vol. 32, no. 3, pp. 1117-1126, Sept. 2017.

[5] A. Ostadi, A. Yazdani, and R. Varma, "Modeling and stability analysis of a DFIG-based wind-power generator interfaced with a series-compensated line," IEEE Trans. Power Del., vol. 24, no. 3, pp. 1504-1514, Oct. 2009.

[6] L. Fan, R. Kavasseri, Z. L. Miao and C. Zhu, "Modeling of DFIG-Based Wind Farms for SSR Analysis," in IEEE Transactions on Power Delivery, vol. 25, no. 4, pp. 2073-2082, Oct. 2010.

[7] R. K. Varma and A. Moharana, "SSR in Double-Cage Induction Generator-Based Wind Farm Connected to Series-Compensated Transmission Line," in IEEE Transactions on Power Systems, vol. 28, no. 3, pp. 25732583, Aug. 2013.

[8] H. A. Mohammadpour and E. Santi, "Modeling and Control of Gate-Controlled Series Capacitor Interfaced With a DFIG-Based Wind Farm," in IEEE Transactions on Industrial Electronics, vol. 62, no. 2, pp. 10221033, Feb. 2015.

[9] J. Sun, "Impedance-based stability criterion for grid-connected inverters," IEEE Trans. Power Electron., vol. 26, no. 11, pp. 3075-3078, Nov. 2011

[10] Z. Miao, "Impedance-Model-Based SSR Analysis for Type 3 Wind Generator and Series-Compensated Network," in IEEE Transactions on Energy Conversion, vol. 27, no. 4, pp. 984-991, Dec. 2012.

[11] I. Vieto and J. Sun, "Damping of subsynchronous resonance involving Type-III wind turbines," in IEEE 16th Workshop on Control and Modeling for Power Electronics (COMPEL), 2015, pp. 1-8.

[12] H. Liu, X. Xie, C. Zhang, Y. Li, H. Liu and Y. Hu, "Quantitative SSR Analysis of Series-Compensated DFIG-Based Wind Farms Using Aggregated RLC Circuit Model," in IEEE Transactions on Power Systems, vol. 
32, no. 1, pp. 474-483, Jan. 2017.

[13] U. Karaagac, J. Mahseredjian, S. Jensen, R. Gagnon, M. Fecteau and I. Kocar, "Safe Operation of DFIG-Based Wind Parks in Series-Compensated Systems," in IEEE Transactions on Power Delivery, vol. 33, no. 2, pp. 709-718, April 2018.

[14] X. Xie, W. Liu, H. Liu, Y. Du and Y. Li, "A System-wide Protection against Unstable SSCI in Series-Compensated Wind Power Systems," in IEEE Transactions on Power Delivery (early access).

[15] Dahai Zhang, Wilsun Xu and A. Nassif, "Flicker source identification by interharmonic power direction," Canadian Conference on Electrical and Computer Engineering, 2005., Saskatoon, Sask., 2005, pp. 549-552.

[16] G. D. Irwin, A. K. Jindal, and A. L. Isaacs, "Sub-synchronous control interactions between type 3 wind turbines and series compensated AC transmission systems," in Proc. IEEE PES General Meeting, 2011, pp.1-6.

[17] "First benchmark model for computer simulation of subsynchronous resonance," in IEEE Transactions on Power Apparatus and Systems, vol. 96, no. 5, pp. 1565-1572, Sept. 1977.

[18] Z. Jin, H. Zhang, F. Shi, Y. Sun and V. Terzija, "A Robust and Adaptive Detection Scheme for Interharmonics in Active Distribution Network," in IEEE Transactions on Power Delivery, vol. 33, no. 5, pp. 2524-2534, Oct. 2018.

[19] J. B. Ekanayake, L. Holdsworth, XueGuang Wu and N. Jenkins, "Dynamic modeling of doubly fed induction generator wind turbines," in IEEE Transactions on Power Systems, vol. 18, no. 2, pp. 803-809, May 2003.

[20] Yazhou Lei, A. Mullane, G. Lightbody and R. Yacamini, "Modeling of the wind turbine with a doubly fed induction generator for grid integration studies," in IEEE Transactions on Energy Conversion, vol. 21, no. 1, pp. 257-264, March 2006.

[21] A. Rolan, A. Luna, G. Vazquez, D. Aguilar and G. Azevedo, "Modeling of a variable speed wind turbine with a Permanent Magnet Synchronous Generator," 2009 IEEE International Symposium on Industrial Electronics, Seoul, 2009, pp. 734-739.

Bo Gao (S'18) obtained the B.Eng degree from Huazhong University of Science and Technology, Wuhan, China and the M.Eng degree from North China Power Electric University, Beijing, China, in 2013 and 2016, respectively. He is currently pursuing the $\mathrm{PhD}$ degree at University of Alberta, Canada. His research interests include power quality and power disturbance analytics.

Yang Wang (M'17) received the B.S degree in electrical engineering from Zhejiang University, Hangzhou, China, in 2012 and Ph.D. degree in electrical and computer engineering at the University of Alberta, Edmonton, AB, Canada, in 2017. From 2017 to 2018, he was a post-doctoral fellow with the University of Alberta, Edmonton, AB, Canada. Currently, he is a research fellow at the College of Electrical Engineering and Information Technology, Sichuan University, China. His main research interests are power quality and integration of renewables.

Wilsun Xu (M'90-SM'95-F'05) obtained the Ph.D. degree in Electrical and Computer Engineering from the University of British Columbia, Vancouver, in 1989. Currently, he is a NSERC/iCORE Industrial Research Chair Professor at the University of Alberta. His current main research interests are power quality and power disturbance analytics.

Guangya Yang (SM'14) received the B.E., M.E., and Ph.D. degrees in 2002, 2005 , and 2008, respectively, all in electric power system. He is currently an Associate Professor in the Center for Electric Power and Energy, Department of Electrical Engineering, Technical University of Denmark. Since 2009, he has been at the Technical University of Denmark, Kongens Lyngby, Denmrk, as a Postdoctoral Researcher and has been leading several industrial collaborative projects in Denmark in the field of monitoring, operation, and protection of renewable energy systems. His research interests include renewable energy integration, smart grids, and cyber-physical energy systems. 